Proceedings

\title{
Urban enclaves and accessibility. The role of walkability in sustainable urban mobility planning
}

\author{
Mara Ladu' ${ }^{1}$, Alessandra Milesi ${ }^{1}$, Ginevra Balletto $^{1}$ and Giuseppe Borruso ${ }^{2}$ \\ 1 DICAAR University of Cagliari; maraladu@hotmail.it; alessandramilesi.unica@gmail.com; \\ ballettoginevra@gmail.com \\ 2 DEAMS University of Trieste; giuseppe.borruso@deams.units.it
}

\begin{abstract}
The process of urban stratification, in its hardware and software components (tangible and intangible) has progressively produced a substantial quantity of buildings, currently in use and disused, which sometimes constitute a real obstacle to urban walkability. The growing attention to sustainability has introduced new contents in urban redevelopment policies and interventions, aimed at favoring higher levels of accessibility (pedestrian and slow in general) in the consolidated urban fabric. The main objective of this study is to define a framework of principles and criteria, to develop a handbook useful to guide the project of new crossings (slow motility) in correspondence of the enclaves, in order to improve urban permeability and walkability, as an integration of the sustainable urban mobility plan.
\end{abstract}

Keywords: urban enclaves; accessibility; walkability

\section{Introduction}

The Covid-19 pandemic has required a review of the accessibility paradigm in the urban mobility system, which refers to the overall change in the position of people and things in space, arose from the need for individuals and companies to consume and use goods and services in places different from those in which they are located. For this reason, it can be said that mobility is the result of a complex phenomenon of economic and social interaction between the various activities distributed over the territory and the transport system. This need is generated by the physical form and the organizational and functional structure of the territory.

Within this framework, if mobility represents the potential ability to move and change in the position, accessibility is the measure of this attitude and represents an interaction between people / goods and activities located in different places. In the last century, Dalvi (1976) [1] defined accessibility as "the degree of ease with which an individual can reach, starting from a spatially separated location, each activity or territorial position using a specific transport system". It means that accessibility depends on four main factors: the characteristics of the individual; the distribution of activities on the territory; the place of departure and arrival; the transport system that connects the reference area to the other areas of the territory.

The ability to achieve high levels of urban accessibility depends on the interaction of different disciplines, including marketing, geography, urban planning, social sciences and transport. In fact, it is possible to decline accessibility in active and passive terms: the first measures the degree of ease with which the subjects (families, businesses) can reach different functions (productive, commercial, social) located in different parts of the territory; the second measures the degree of ease with which the functions located in a specific place can be reached by users that come from different parts of the territory. 
Until the 1990s, accessibility was linked to a dual concept that led to the recognition of the transport system as the main response to the demand for local mobility. From the 90 s onwards, web technology and, in general, ICT systems, have led to major changes in the ways of relating and using activities in the territories, transforming the consolidated dual paradigm (spatial proximity-physical mobility) into a tripolar system (spatial proximity-physical mobility-digital connection).

The pandemic has made the value of digital connection systems even more evident in guaranteeing adequate accessibility to primary services and beyond. At the same time, the global aim of guaranteeing an inclusive, safe, healthy and sustainable city has brought attention back to open space and sustainable and slow urban mobility, including pedestrian mobility. In fact, the health crisis has highlighted the need of people to move on foot to reach the main urban functions [2], making walkability a transversal element of the tripolar system (Fig. 1).

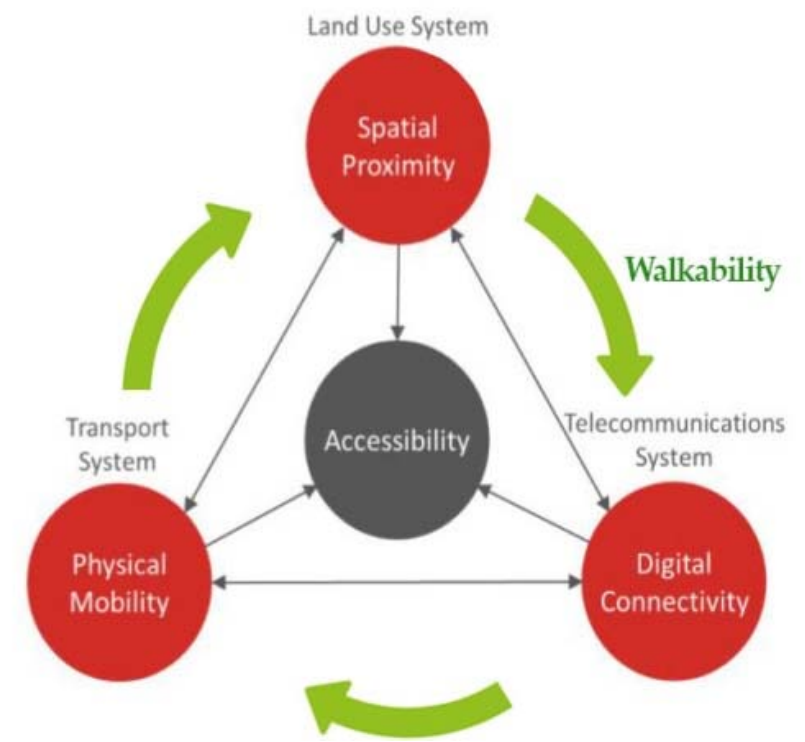

Figure 1. The walkability as a transversal element of the tripolar system (spatial proximityphysical mobility-digital connection).

In Europe, the Sustainable Urban Mobility Plan (SUMP) is developed on these assumptions and represents an important planning tool to support the construction of a Proximity City, which guarantees higher levels of pedestrian accessibility in the urban environment.

\section{Walkability and Sustainable urban mobility}

In the Italian context, the Sustainable Urban Mobility Plan (SUMP) is a development tool based on the principles of integration, participation, monitoring and evaluation for the mobility demand of people and goods with the aim of improving the quality of life. In particular, the SUMP aims to improve the quality and environmental performance of urban areas in order to guarantee a healthier urban system within an overall framework of economic and social sustainability, guaranteeing the right to mobility, without gravity, as far as possible, on the community, in terms of air and noise pollution, traffic and accidents. Active urban mobility, including walking and cycling, contributes to the construction of healthier and socially inclusive cities. The concept of walkability is linked to that of quality of life and depends on the presence of public spaces and urban facilities and their spatial distribution. In these terms it becomes part of the SUMP, without however acquiring the status of a real system of sustainable urban mobility, although it has effects on improving mood, self-esteem and personal balance 
[3]. The SUMP represents the cognitive framework to develop networks of pedestrian 1 paths, transforming the presence of urban enclaves into opportunities, also by modifying the existing morphology. In fact, the creation of new pedestrian paths into the enclaves guarantees a higher proximity of urban facilities located in different parts of the city, thus favoring walkability, in line with the 15-minute city paradigm [4,5].

\section{Methodology}

According to the need to develop a "Proximity City", the authors define a series of indicators to guide the creation of new pedestrian paths within the urban enclaves and to evaluate its positive externalities [6]. Four indicators have been defined, expression of the characteristics of the single building or public real estate complex (intrinsic characteristics), and of those of its context (extrinsic characteristics):

1) attractiveness (A): the number and variety of boundary functions reachable in 15 minutes on foot (the reference is to the Simpson diversity index [7]);

2) uniqueness (Un): the number of the possible crossings into the enclaves with respect to the existing ones in the neighboring context;

3) usability (Us): the degree of difficulty in walking the crossing, determined by the physical characteristics of the space, whether it is open or closed;

4) satisfaction (S): the number of inhabitants who gravitate to the neighborhood and who would benefit from the opening of the crossing;

5) feasibility (F): the technical and economic possibility of making the crossing.

The Proximity Index (PI) is given by the sum of these indicators, appropriately weighted, according to the following formula:

$\mathrm{PI}=\sum_{i=1}^{n}\left(I_{i} * p_{k}\right) * 100$ where $\sum_{k=1}^{n} p_{k}=1$ and Ii $=\mathrm{A}, \mathrm{Un}, \mathrm{Us}, \mathrm{S}, \mathrm{F} \ldots$ where $\mathrm{i}=1,2,3, . . \mathrm{n}$

where $p_{k}$ represents the weight attributed to each index.

\section{Results and conclusions}

The calculation of the Proximity Index (PI) provides a preliminary assessment of the degree of transformability of the enclaves in terms of pedestrian crossing. In fact, this index represents a useful tool to support the decision-making process in the implementation of urban regeneration projects aimed at developing a Proximity City. In particular, it assigns a different degree of priority of intervention to each part of the city considered in the evaluation, having the characteristics of an enclave. Moreover, this index also aims to be a stimulus for the introduction, in urban governance, of temporary interventions. In particular, the feasibility indicator informs about the possibility of creating new pedestrian crossings in urban enclaves, even before carrying out an overall redevelopment project of the building complex. In many urban situations, such temporary interventions aim at promoting urban walkability, increase the attractiveness of the property, give it a new identity and facilitate the choice of new uses.

\section{References}

1. Dalvi, M.Q.; Martin, K.M. The measurement of accessibility: some preliminary results. Transportation 1976, 5(1), 17-42.

2. Campisi, T.; Ignaccolo, M.; Inturri, G.; Tesoriere, G.; Torrisi, V. Evaluation of walkability and mobility requirements of visually impaired people in urban spaces. Research in Transportation Business \& Management 2021, 40, 100592

3. Balletto, G.; Pezzagno M.; Richiedei, A. 15-Minute City in Urban Regeneration Perspective: Two Methodological Approaches Compared to Support Decisions. In International Conference on Computational Science and Its Applications; Springer: Cham, 2021; pp. 535-548 
4. Scorza, F.; Fortunato, G.; Carbone, R.; Murgante, B.; Pontrandolfi, P. Increasing Urban Walkability through 1 Citizens' Participation Processes. Sustainability 2021, 13(11), 5835.

5. Meng, L. I. The planning strategies of a 15-minute community life circle based on behaviors of residents. Urban 3 Planning Forum 2017, 111-118

6. Balletto, G.; Ladu, M.; Milesi, A.; Borruso, G. Methodological Approach on Disused Public Properties in the 15- 5 Minute City Perspective. Sustainability 2021, 13(2), 593.

7. Borruso, G.; Porceddu, A. A tale of two cities: Density analysis of CBD on two midsize urban areas in Northeastern Italy. In Geocomputation and Urban Planning. Studies in Computational Intelligence; Murgante, B., Borruso, G., Lapucci, A., Eds.; Springer: Berlin/Heidelberg, Germany, 2009; Volume 176. 